\title{
Modulation of the caveolin-3 localization to caveolae and STAT3 to mitochondria by catecholamine-induced cardiac hypertrophy in $\mathrm{H} 9 \mathrm{c} 2$ cardiomyoblasts
}

\author{
Kyuho Jeong*, Hayeong Kwon*, \\ Chanhee Min and Yunbae Pak ${ }^{1}$ \\ Department of Biochemistry \\ Division of Applied Life Science (BK21), PMBBRC \\ Environmental Biotechnology National Core Research Center \\ Gyeongsang National University \\ Jinju 660-701, Korea \\ ${ }^{1}$ Corresponding author: Tel, 82-55-751-5961; \\ Fax, 82-55-759-9363; E-mail, ybpak@nongae.gsnu.ac.kr \\ *These authors contributed equally to this work. \\ DOI 10.3858/emm.2009.41.4.025
}

\section{Accepted 20 November 2008}

Abbreviations: Akt, protein kinase B; CsA, cyclosporin A; GPCR, $G$ protein-coupled receptor; ISO, isoproterenol; $\mathrm{PE}$, phenylephrine; RTK, receptor tyrosine kinase; STAT3, signal transducers and activator of transcription 3

\begin{abstract}
We investigated the effect of phenylephrine (PE)- and isoproterenol (ISO)-induced cardiac hypertrophy on subcellular localization and expression of caveolin-3 and STAT3 in H9c2 cardiomyoblast cells. Caveolin-3 localization to plasma membrane was attenuated and localization of caveolin-3 to caveolae in the plasma membrane was $24.3 \%$ reduced by the catecholamineinduced hypertrophy. STAT3 and phospho-STAT3 were up-regulated but verapamil and cyclosporin A synergistically decreased the STAT3 and phosphoSTAT3 levels in PE- and ISO-induced hypertrophic cells. Both expression and activation of STAT3 were increased in the nucleus by the hypertrophy. Immunofluorescence analysis revealed that the catecholamineinduced hypertrophy promoted nuclear localization of pY705-STAT3. Of interest, phosphorylation of pS727STAT3 in mitochondria was significantly reduced by catecholamine-induced hypertrophy. In addition, mitochondrial complexes II and III were greatly downregulated in the hypertrophic cells. Our data suggest that the alterations in nuclear and mitochondrial activation of STAT3 and caveolae localization of caveolin-3 are related to the development of the catechol-
\end{abstract}

amine-induced cardiac hypertrophy.

Keywords: cardiomegaly; caveolae; caveolin-3; cell nucleus; heart; isoproterenol; mitochondria; phenylephrine; STAT3 transcription factor

\section{Introduction}

Hypertension is major risk factors for cardiac damage, ischemia, myocardial infarction, and congestive heart failure (Zampaglione et al., 1996). In response to increased demands for cardiac work caused by various pathologic stresses, heart adapts through compensatory hypertrophy of myocytes. Thus, cardiac hypertrophy is recognized in many cardiovascular diseases, such as hypertension, vascular disease, and myocardial infarction, and is an independent risk factor for cardiac morbidity and mortality. Hypertrophic stimuli induce an increase in cell size in the absence of cell division through $\mathrm{Ca}^{2+}$ signaling and activation of $\mathrm{PKC}$, MAPK and PKB/ Akt (Watanabe et al., 2001; Dorn and Force, 2005), and are accompanied by increased protein synthesis with reprogramming of gene expression (Takeo et al., 2000). Cardiac hypertrophy is induced by a variety of factors, such as vasoactive peptides, growth factors, cytokines, and hormones (Nicol et al., 2001). Catecholamines, including phenylephrine (PE) and isoproterenol (ISO) play pivotal roles in cellular growth (Colombo et al., 2001) and induce cardiac hypertrophy (Taigen et al., 2000; Zou et al., 2001; Hwang et al., 2006). Persistent stimulation of cardiac cells by catecholamine has been known to induce activation of the $\mathrm{Ca}^{2+}$ /calmodulin-dependent phosphatase, calcineurin and described as a prime example of calcineurin-induced cardiac hypertrophy (Karpen and Rich, 2001; Zou et al., 2001).

Caveolin is a principal protein component of caveolae (Anderson, 1998; Smart et al., 1999; Razani et al., 2002). It has been proposed that caveolin family members function as scaffolding proteins (Schlegel et al., 1999) to organize lipid-modified signaling molecules (G-proteins, Src-family kinase, PKC $\alpha$, and eNOS) (Segal et al., 1999; Everson and Smart, 2001; Li et al., 2001; Oh and Schnitzer, 2001). Caveolin also directly interacts with many 
growth factor receptors (RTKs, EGFR, PDGF and VEGF), leading to the inhibition of their function (Couet et al., 1997; Liu et al., 1999; Yamamoto et al., 1999). Caveolin-3 is known as a muscle-specific membrane protein crucial for myoblast differentiation. The expression of caveolin-3 is linked to the maturation of muscle phenotype and it is tightly regulated by hypertrophic $\mathrm{C} 2 \mathrm{C} 12$ myoblast cells (Fanzani et al., 2007). Signal transducer and activator of transcription factor 3 (STAT3) is a critical mediator for survival of cardiomyocytes (Sano et al., 2000) and appears to be essential in the induction of cardiac myocyte hypertrophy through gp130 (Kunisada et al., 1998; Kunisada et al., 2000). The underlying physiological mechanisms of caveolin-3 and STAT3 in cardiac myocyte hypertrophy, however, have not yet been elucidated.

The present study was conducted to investigate whether caveolin-3 and STAT3 as regulatory molecules contribute to the hypertrophy of cardiac cells in an experimental model of catecholamine-induced hypertrophic H9c2 cardiomyoblast cells. We generated the hypertrophic $\mathrm{H} 9 \mathrm{c} 2$ cardiomyoblasts by $\mathrm{PE}$ and ISO treatment and examined the hypertrophy along with specific inhibitors, verapamil and cyclosporin A (CsA). By subcellular and caveolinrich membrane fractionations and immunofluo- rescence microscopy, our data indicate that the alternation of caveolin-3 and STAT3 status is linked to the development of cardiac hypertrophy.

\section{Results}

\section{Expression of caveolin-3 in catecholamine-induced hypertrophy}

To verify the catecholamine-induced cellular hypertrophy in $\mathrm{H} 9 \mathrm{c} 2$ cardiomyoblasts, the cell surface area measurement was performed as described in Methods. As demonstrated in Figure 1, in $\mathrm{H} 9 \mathrm{c} 2$ cells treated with PE or ISO, cell size was significantly increased by 2.2 or 2.3 fold, respectively.

The effect of catecholamine-induced hypertrophy on expression of caveolins and hypertrophyrelated intracellular signal molecules and their activation was examined in $\mathrm{H} 9 \mathrm{c} 2$ cardiomyoblast cells. When the hypertrophy was induced by PE and ISO treatment, the expression of caveolin-3, caveolin-2, calcineurin, ERK, and Akt showed no detectable changes in the hypertrophic cells as compared to the untreated cells (Figure 2A). Phosphorylation of ERK and Akt also showed no changes by PE- or ISO-induced hypertrophy. These
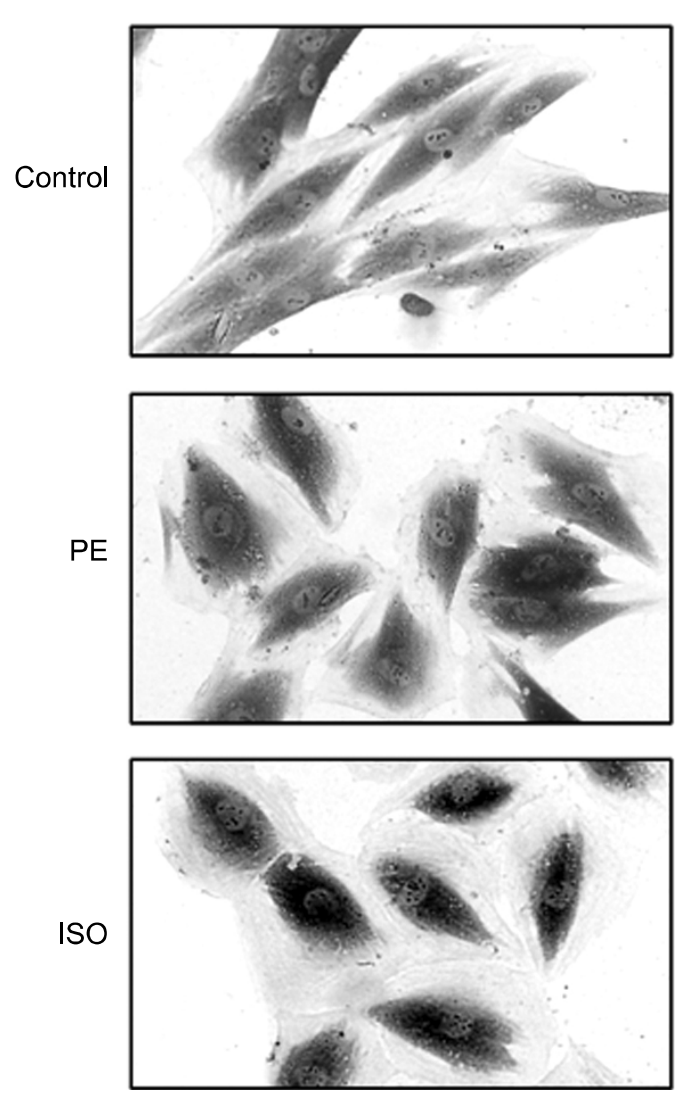

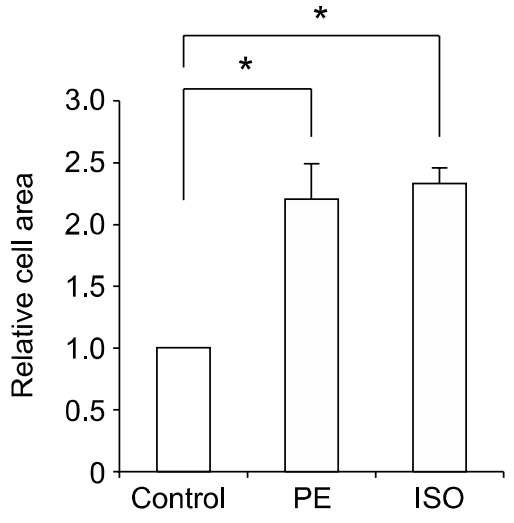

Figure 1. Morphological changes and measurement of hypertrophic growth of $\mathrm{H} 9 \mathrm{c} 2$ cardiac myocytes by PE- or ISO-induced hypertrophy. $\mathrm{H} 9 \mathrm{c} 2$ cells were maintained in $1 \%$ serum media for $18 \mathrm{~h}$ and treated with PE $(50 \mu \mathrm{M})$ or ISO $(10 \mu \mathrm{M})$ for $48 \mathrm{~h}$. The cellular hypertrophy was assayed by cell surface area measurement. Cell size was analyzed using Image Pro Plus software, and the values represent the relative area \pm S.E., $n=3 .{ }^{*}, P<$ 0.01 . 
A

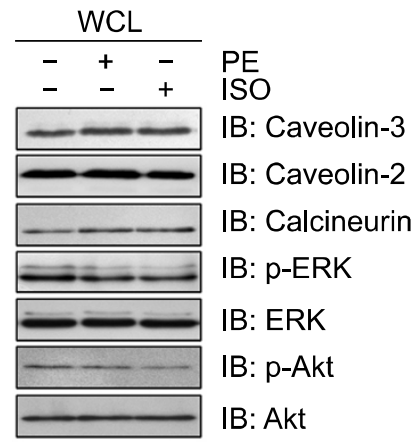

B

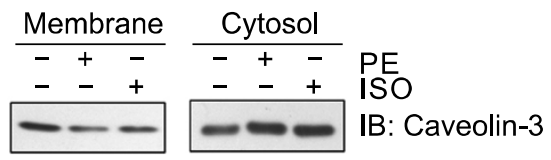

C
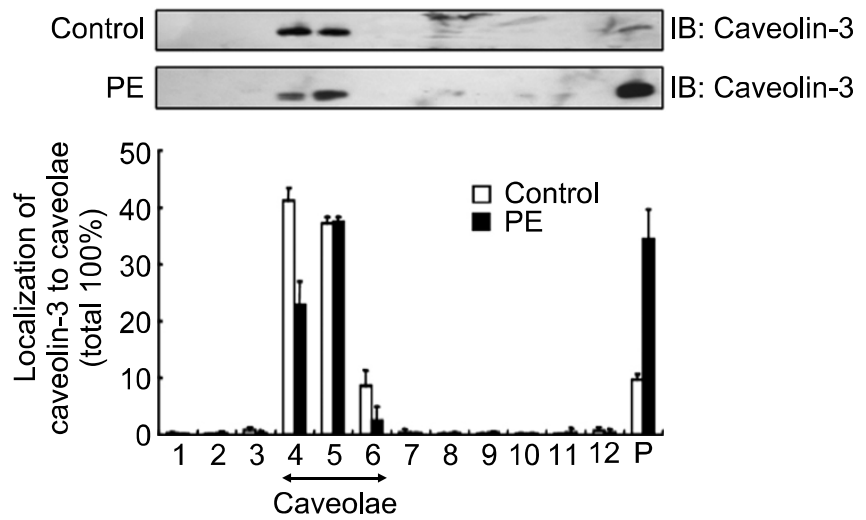

D

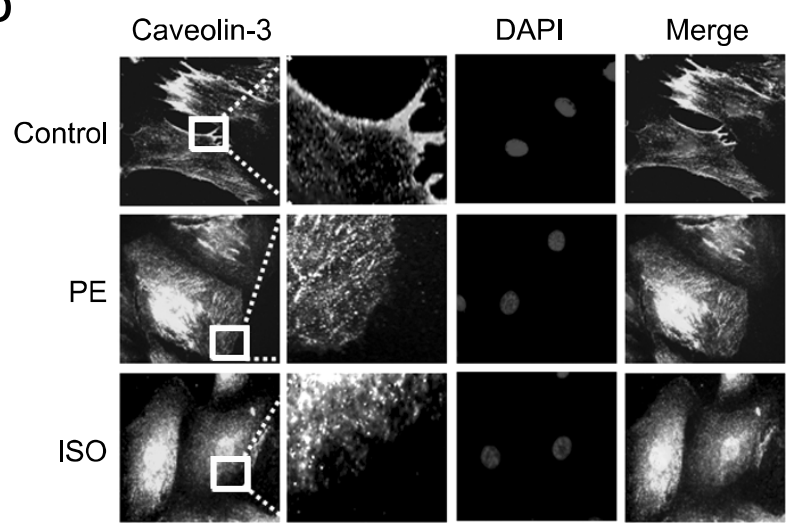

Figure 2. Effect of catecholamine-induced hypertrophy on expression and cellular localization of caveolin-3. (A) Cells were maintained in $1 \%$ serum media for $18 \mathrm{~h}$ and treated with PE $(50 \mu \mathrm{M})$ or ISO $(10 \mu \mathrm{M})$ for $48 \mathrm{~h}$. Whole cell lysates $(\mathrm{WCL})$ were subjected to immunoblot analysis using anti-caveolin-3, anti-caveolin-2, anti-calcineurin, anti-phospho-ERK, anti-ERK, anti-phospho-Akt, and anti-Akt antibodies. (B) Cells were incubated with PE (50 $\mu$ M) or ISO $(10 \mu \mathrm{M})$ for $48 \mathrm{~h}$ and subjected to subcellular fractionation as described in "Methods". Membrane and cytosol fractions were subjected to SDS-PAGE and then immunoblot analysis with anti-caveolin-3 antibody. (C) Cells were incubated with PE (50 $\mu \mathrm{M})$ for $48 \mathrm{~h}$. Isolation of caveolae were performed by sucrose density fractionation. Fractions collected were subjected to immunoblot analysis with anti-caveolin-3 antibody. Distribution of caveolin-3 was quantified by densitometry. The results represent mean \pm S.E. of three independent experiments. (D) H9c2 cells were plated onto coverslips and maintained in $1 \%$ serum media for $18 \mathrm{~h}$. Cells were then incubated with PE $(50 \mu \mathrm{M})$ or ISO $(10 \mu \mathrm{M})$ for $48 \mathrm{~h}$. After fixation and permeabilization, cells were stained with anti-caveolin-3-antibody followed by Alexa Fluor ${ }^{R} 488$-conjugated antibody, respectively as described under "Methods". Coverslips were mounted on a slide and analyzed by fluorescence microscopy. Results are representative images of cells from three independent experiments. Green: Caveolin-3, Blue: Nucleus (DAPI), Merge: Caveolin-3 + DAPI.

data indicate that the catecholamine-induced hypertrophy has no effect on the caveolin-3 expression.

\section{Caveolae localization of caveolin- 3 in catecholamine-induced hypertrophy}

Interestingly, when we preliminarily tested any retardation in membrane association of caveolin-3 in catecholamine-induced hypertrophic cells, PEand ISO-induced hypertrophy resulted 41.4 and $30 \%$ reduction in the membrane localization of caveolin-3, respectively (Figure 2B). Since these results suggested that recruitment of caveolin-3 to the plasma membrane-containing membrane, more specifically to caveolae might be retarded by the catecholamine-induced hypertrophy, we investigated further the effect of the PE- or ISO-induced hypertrophy on caveolae localization of caveolin-3. As caveolin-rich membrane fractions were analyzed as shown in Figure 2C, caveolae were isolated from most cellular proteins separated in non caveolar fractions 9-12 and a pellet, and localized in fractions 4, 5 and 6 in the sucrose gradient fractionation. The caveolae localization was $24.3 \%$ reduced by $\mathrm{PE}$-induced hypertrophy with a consequent increase in the bottom phase of pellet in non caveolar fraction. The results were further confirmed by immunofluorescence microscopy to directly assess the subcellular localization of caveolin-3. 
Consistent with the result shown in Figure $2 \mathrm{C}$, caveolin-3 localized to the plasma membrane in untreated cells (Figure 2D, panel 1). The localization of caveolin-3 was, however, prevented in PEand ISO-induced hypertrophic cells (Figure 2D, panels 2 and 3 ). Thus, these results demonstrate that the catecholamine-induced hypertrophy attenuates the localization of caveolin-3 to caveolae in the plasma membrane.

\section{Modulation in expression and activation of STAT3 by catecholamine-induced hypertrophy}

STAT3 is known to play a critical role in cardiac hypertrophy (Kunisada et al., 1998, 2000). Recently, ISO-induced phosphorylation of pY705-STAT3 was reported in neonatal rat cardiomyocytes (Zhang et al., 2008). When we examined the expression and phosphorylation of STAT3 in the PE- and ISOinduced hypertrophic $\mathrm{H} 9 \mathrm{c} 2$ cardiomyoblasts, phosphorylation of pY705- STAT3 was dramatically increased by 16.2 and 19.8 fold, respectively (Figure 3, panel 1, lanes 2 and 3). Phosphorylation of pS727-STAT3 was increased by 1.8 fold in both PE- and ISO-induced hypertrophic cells as compared to the untreated cells (Figure 3, panel 2, lanes 2 and 3). STAT3 was 1.6 fold up-regulated in the hypertrophic cells (Figure 3 , panel 3, lanes 2 and 3). STAT1, unlike STAT3 showed no changes (Figure 3, panel 4). These data show that expression and activation of STAT3 was up-regulated by the catecholamine- induced hypertrophy.

Verapamil, a $\mathrm{Ca}^{2+}$ channel inhibitor and CsA, a calcineurin inhibitor have been known to prevent

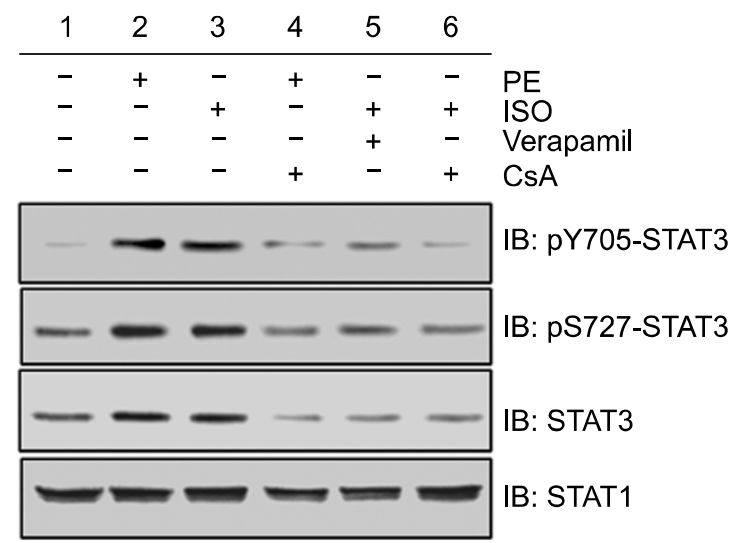

Figure 3. Effect of catecholamine-induced hypertrophy on expression and activation of STAT3. Cells were treated as follows lanes; (1), control; (2), PE $(50 \mu \mathrm{M})$ for $48 \mathrm{~h}$; (3), ISO $(10 \mu \mathrm{M})$ for $48 \mathrm{~h}$; (4), PE $(50 \mu \mathrm{M})$ plus $\mathrm{CsA}(500 \mathrm{ng} / \mathrm{ml})$ for $48 \mathrm{~h}$; (5), ISO $(10 \mu \mathrm{M})$ plus verapamil $(1 \mu \mathrm{M})$ for 48 $\mathrm{h}$; (6), ISO $(10 \mu \mathrm{M})$ plus CsA (500 ng/ml) for $48 \mathrm{~h}$. WCL were subjected to immunoblot analysis with anti-STAT3, anti-pS727-STAT3, anti-pY705STAT3, and anti-STAT1 antibodies. cardiac hypertrophy in various rodent models induced by PE or ISO treatment (Kato et al., 2000; Taigen et al., 2000; Zou et al., 2001). When PE- or ISO-induced hypertrophic $\mathrm{H} 9 \mathrm{c} 2$ cardiomyoblast cells were treated with verapamil or CsA, STAT3 was down-regulated below the basal level (Figure 3, panel 3, lanes 4, 5, and 6). Phosphorylation of pY705- and pS727-STAT3 was also markedly reduced by verapamil or CsA as compared to the untreated cells (Figure 3, panels 1 and 2, lanes 4, 5 , and 6 ). These results show that activation of STAT3 is up-regulated by the catecholamine-induced cardiac hypertrophy through $\mathrm{Ca}^{2+}$-dependent signaling pathway in $\mathrm{H} 9 \mathrm{c} 2$ cardiomyoblasts.

\section{Alteration of STAT3 activation in mitochondria and nucleus by catecholamine-induced hypertrophy}

A recent study showed that STAT3 activation is required for maintaining mitochondrial complex function of the respiratory chain in primary pro-B lymphocytes (Potla et al., 2006). We investigated the effect of catecholamine-induced hypertrophy on expression and phosphorylation of STAT3 in mitochondria and nucleus. In untreated cells, 38.8 $\pm 5 \%(n=3)$ of pS727-STAT3 was detected in mitochondrial fraction. However, the mitochondrial activation of pS727-STAT3 was reduced to $27 \pm$ $3 \%(n=3)$ and $25.6 \pm 3 \%(n=3)$ in PE- and ISOinduced hypertrophic cells, respectively (Figure 4A, panel 1, a). No significant changes were detected on STAT3 expression and activation of pY705STAT3 in mitochondrial fraction of the hypertrophic cells (Figure 4A, panels 2 and $3, b$ and $c$ ). Of interest, the expression and phosphorylation of STAT3 in the nucleus were increased by PE- and ISO-induced hypertrophy (Figure 4A, panels 1, 2, and $3, a, b$, and $c$ ). No significant changes were detected in nuclear and mitochondrial localization of STAT1 by catecholamine-induced hypertrophy (Figure 4A, panel 4, d). In support of the fractionation data, immunofluorescence staining demonstrated the nuclear translocation of pY705-STAT3 in response to $\mathrm{PE}$ and ISO treatment as compared to the untreated cells (Figure 4B). Taken together, these results indicate that the catecholamine-induced hypertrophy modulates the expression and activation of STAT3 in mitochondria and nucleus.

\section{Down-regulation of mitochondria complexes by catecholamine-induced hypertrophy}

The oxidative phosphorylation system is located in the inner membrane of mitochondria and comprises five individual complexes (complexes I to V) (Schägger and Pfeiffer, 2000). Since the phospho- 


\section{A}

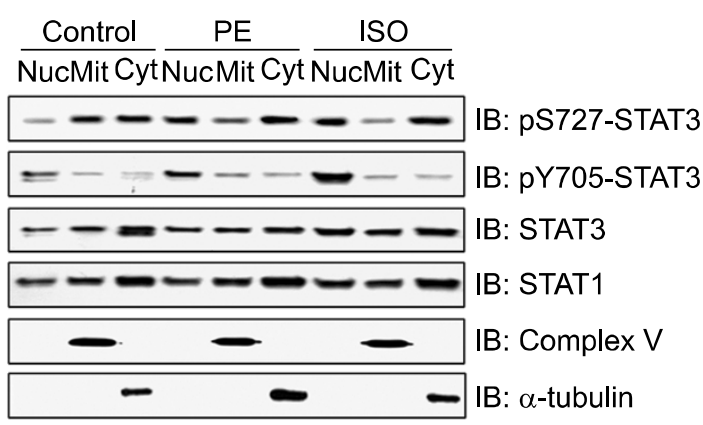

a

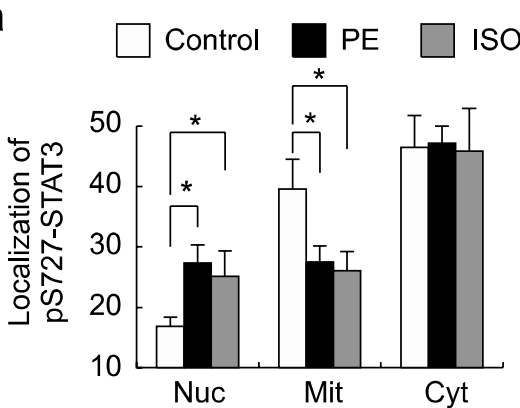

C

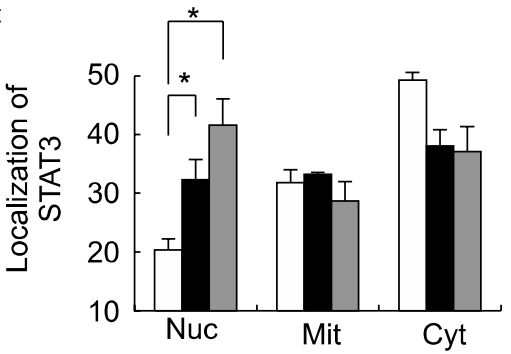

b

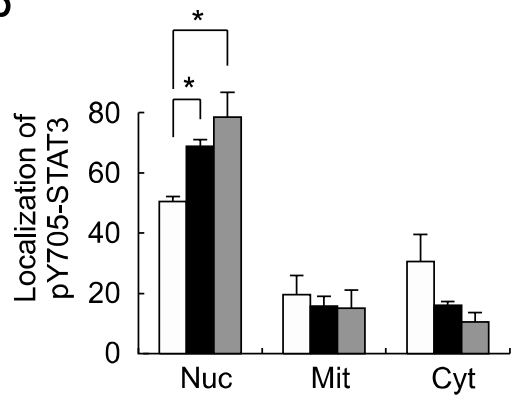

d

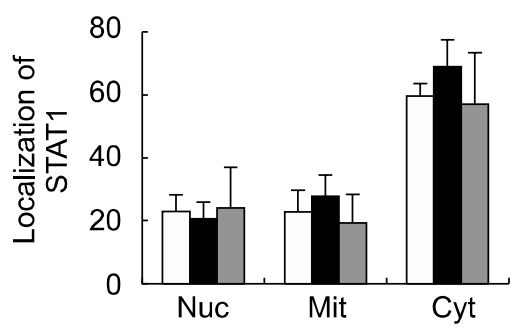

B

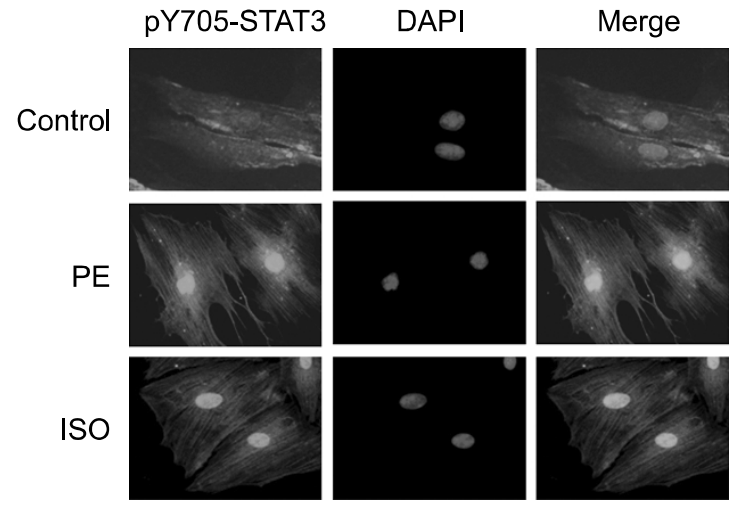

Figure 4. Effect of catecholamine-induced hypertrophy on expression and activation of STAT3 in mitochondria and nucleus. H9c2 cells were maintained in $1 \%$ serum media for $18 \mathrm{~h}$. Cells were then incubated with PE $(50 \mu \mathrm{M})$ or ISO $(10 \mu \mathrm{M})$ for $48 \mathrm{~h}$. (A) Cytosolic (Cyt), nuclear (Nuc), and mitochondrial (Mit) fractions were analyzed by immunoblotting using antibodies specific for STAT3, pS727-STAT3, pY705-STAT3, STAT1, anti-complex V, anti- $\alpha$-tubulin. Subcellular localization of STAT3 and phosphorylation of STAT3 were quantified by densitometry. The protein levels were quantified by densitometry and the sum of Cyt, Nuc, and Mit fractions set arbitrarily to $100 \%$. Quantification represents the relative intensity to the control cells; mean $\pm S$.E., $n=3$. ${ }^{*}, P$ $<0.05$ (a-d). (B) H9c2 cells were plated onto coverslips and maintained in $1 \%$ serum media for $18 \mathrm{~h}$. Cells were then incubated with PE (50 $\mu \mathrm{M})$ or ISO $(10 \mu \mathrm{M})$ for $48 \mathrm{~h}$. After fixation and permeabilization, cells were stained with anti-pY705-STAT3 antibody followed by TRITC-conjugated antibody, respectively as described under "Methods". Coverslips were mounted on a slide and analyzed by fluorescence microscopy. Results are representative images of cells from three independent experiments. Red: pY705-STAT3; Blue: Nucleus (DAPI); Merge: pY705-STAT3 + DAPI. 


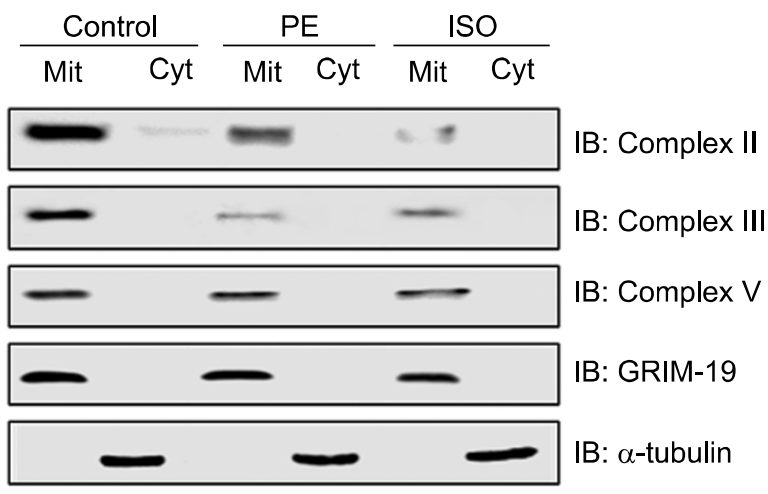

Figure 5. Effect of catecholamine-induced hypertrophy on expression of mitochondria complexes. $\mathrm{H} 9 \mathrm{c} 2$ cells were maintained in $1 \%$ serum media for $18 \mathrm{~h}$ and incubated with PE $(50 \mu \mathrm{M})$ or ISO $(10 \mu \mathrm{M})$ for $48 \mathrm{~h}$. WCL were subjected to mitochondrial fractionation. Equal amounts of protein from cytosolic and mitochondrial fractions were immunoblotted with anti-complex II, anti-complex III, anti-complex V, anti-GRIM-19 and anti- $\alpha$-tubulin antibodies.

rylation of pS727-STAT3 in the mitochondria is impaired by the PE- and ISO-induced hypertrophy (Figure 4A), we further explored the effect of PEand ISO-induced hypertrophy on expression of the mitochondria complexes. As shown in Figure 5, complex II and III was 34 and $81 \%$ down-regulated in PE- and 59 and $41 \%$ in ISO-induced hypertrophic cells, respectively with no changes in complex V. Collectively, these data suggest that the PE- and ISO-induced hypertrophy decreases activation of pS727-STAT3 in mitochondria and in turn, attenuates mitochondria function to carry on oxidative phosphorylation, possibly by preventing the interaction between pS727-STAT3 and mitochondrial complexes.

\section{Discussion}

We investigated the regulatory function of caveolin-3 and STAT3 in catecholamine-induced cardiac hypertrophy. It has been shown that the expression of caveolin-3 is increased in hypertrophied $\mathrm{C} 2 \mathrm{C} 12$ myoblasts transfected with an activated form of Akt (Fanzani et al., 2007). In the present study with $\mathrm{H} 9 \mathrm{c} 2$ cardiomyoblasts, the PE- or ISO-induced hypertrophy exhibited no changes on the activation of Akt and expression of caveolin-3 (Figure 2A). Although it would appear that caveolin-3 in the catecholamine-induced hypertrophic cells is not directly involved in PI3-kinase/Akt-regulated signaling pathway, our data show that the hypertrophy caused retardation of caveolin-3 translocation to caveolae in plasma membrane from cytosol (Figure $2 \mathrm{C}$ and $\mathrm{D}$ ). These results indicate a possible defect in the caveolae localization of caveolin-3 in development of the catecholamine-induced cardiomyoblast hypertrophy.

STAT3 signaling pathway has been shown to mediate cardiac hypertrophy in fetal cardiomyocytes and transgenic mice (Kunisada et al., 1998, 2000). Recent study with neonatal rat cardiomyocytes demonstrated that ISO increases tyrosine phosphorylation of STAT3 (Zhang et al., 2008). As we investigated the effect of PE- and ISO-induced hypertrophy on activation of STAT3 in H9c2 cardiomyocytes, the phosphorylation of pY705- and pS727-STAT3 was increased by the catecholamine-induced cardiac hypertrophy (Figure 3). When we further examined effects of the specific inhibitors, verapamil and CsA in order to define the regulatory mechanism by STAT3, verapamil and CsA prevented the catecholamine-induced expression and activation of STAT3 (Figure 3), suggesting an involvement of the $\mathrm{Ca}^{2+}$ - dependent phosphatase calcineurin in the catecholamine-induced cardiac hypertrophy. Thus, our findings indicate that phospho-STAT3 has a regulatory role in the catecholamine-induced development of cardiomyoblast hypertrophy.

STAT3 has been reported to interact with GRIM19 , known component of mitochondrial complex I (Lufei et al., 2003; Zhang et al., 2003; Potla et al., 2006). However, the molecular mechanisms by which STAT3 regulates mitochondrial function in cardiac hypertrophy have not been established. The present study demonstrates that phosphorylation of pS727-STAT3 in mitochondria is attenuated by PE- and ISO-induced cardiac hypertrophy (Figure 4A, a). Further, the mitochondrial complexes in mitochondria oxidative phosphorylation system were remarkably down-regulated by the hypertrophy (Figure 5). These results suggest that reduction of pS727-STAT3 activation in the mitochondria leads to the down-regulation of mitochondrial complexes in response to catecholamine-induced hypertrophy. In contrast, the expression and activation of STAT3 in nucleus were increased by the PE- and ISO-induced hypertrophy (Figure 4A, a, b and c). Re-localization of pY705STAT3 to the nucleus was also observed in catecholamine-induced hypertrophic cells (Figure 4B). Thus, these results suggest that the increased activation of STAT3 in the nucleus leads to transactivation of hypertrophic responsible genes such as ANF and c-fos as described (Kunisada et al., 1998).

In summary, the present study provides evidence that the catecholamine-induced cardiomyoblast hypertrophy in H9c2 cells attenuates the translocation of caveolin-3 to caveolae in plasma membrane, promotes nuclear localization of pY705- 
STAT3, increases expression and activation of STAT3 in nucleus, and reduces pS727-STAT3 activation in mitochondria. Thus, the modulation of caveolin-3 and STAT3 status by the hypertrophy play an important role in the pathogenesis of cardiomyoblast hypertrophy.

\section{Methods}

\section{Cell culture and treatment}

$\mathrm{H} 9 \mathrm{c} 2$ (a cardiomyoblast cell line derived from embryonic rat heart tissue) cells were maintained in DMEM (GIBCO/BRL) supplemented with 10\% FBS (Hyclone, Logan, Utah) and $100 \mathrm{U} / \mathrm{ml}$ penicillin/streptomycin (Sigma Chemical Co., St. Louis, $\mathrm{MO}$ ) in a $5 \% \mathrm{CO}_{2}$ incubator at $37^{\circ} \mathrm{C}$ as described previously (Ha and Pak, 2005; Kwon et al., 2008). To test an experimental hypertrophy condition, cells were serum starved for $18 \mathrm{~h}$ in DMEM containing $1 \%$ FBS and treated with either $50 \mu \mathrm{M}$ PE or $10 \mu \mathrm{M}$ ISO for 48 $h$. To investigate the effect of selective inhibitors in the catecholamine-induced hypertrophy, cells were treated with either $1 \mu \mathrm{M}$ verapamil or $500 \mathrm{ng} / \mathrm{ml}$ CsA for $48 \mathrm{~h}$ together with PE or ISO (Taigen et al., 2000). PE, ISO, verapamil, and CsA were obtained from Sigma Chemical Co. (St. Louis, MO).

\section{Measurement of hypertrophic growth in $\mathrm{H} 9 \mathrm{c} 2$ cardiomyoblasts}

Hypertrophy was assayed by measurement of cell surface area of H9c2 cells as previously described (Hwang et al., 2006; Alvarez et al., 2007). Cells grown on glass coverslips were serum starved for $18 \mathrm{~h}$ in DMEM containing $1 \%$ FBS and treated with either PE or ISO for $48 \mathrm{~h}$. The cells were washed with PBS and fixed for 20 min at RT with $3.7 \%$ paraformaldehyde in PBS. Fixed cells were rinsed with PBS and stained with $0.1 \%$ Crystal violet (Sigma) for 10 min. The coverslips were then washed and mounted on glass slides. Images were obtained using an Olympus DP-71 digital camera attached to an Olympus BX51 microscope. Ten random photographs were taken from each sample and surface area from at least five cells from each photograph was determined using Image Pro Plus 6.1 software (MediaCybernetics). The data shown represent the image analysis from three independent experiments.

\section{Immunoblot analysis}

Proteins extracts were generated using RIPA buffer $(50 \mathrm{mM}$ HEPES, $150 \mathrm{mM} \mathrm{NaCl}, 100 \mathrm{mM}$ Tris- $\mathrm{HCl}, \mathrm{pH} 8.0,1 \%$ Triton $\mathrm{X}-100,1 \%$ deoxycholic acid, $0.1 \%$ SDS, $5 \mathrm{mM}$ EDTA) containing $10 \mathrm{mM} \mathrm{NaF}, 5 \mathrm{mM}$ DTT, $1 \mathrm{mM}$ PMSF, $1 \mathrm{mM}$ sodium vanadate, $20 \mu \mathrm{M}$ leupeptin, and $100 \mu \mathrm{M}$ aprotinin) as described before (Kim and Pak, 2005). Equal amounts of samples $(50 \mu \mathrm{g})$ were separated on 10 or $12.5 \%(\mathrm{w} / \mathrm{v})$ SDS-polyacrylamide gels and transferred to PVDF membrane (Millipore; Bedford, MA). Transfers were blocked overnight at $4^{\circ} \mathrm{C}$ with $5 \%(\mathrm{v} / \mathrm{v})$ nonfat dry milk in TBS, $0.1 \%$ $(\mathrm{v} / \mathrm{v})$ Tween 20, and then incubated for $2 \mathrm{~h}$ at room temperature (RT) in the primary antibody. The primary antibodies used were as follows: caveolin-2 (BD 610658), caveolin-3 (BD 610420), ERK (BD 610031), Akt (BD 610877), calcineurin (BD 610259), STAT3 (BD 610190), and GRIM-19 (BD 612388) antibodies from BD Transduction Laboratories; $\alpha$-tubulin (sc-5286) antibody from Santa Cruz Biotechnology; phospho-ERK (Thr202 and Tyr204) (\#9101), phospho-Akt (Ser473) (\#9271), pS727STAT3 (\#9136), and pY705- STAT3 (\#9135) antibodies from Cell Signalling; complex II (MS204), complex III (MS304), and complex $\mathrm{V}$ (MS507) antibodies from Mito Science. The membrane were washed with TBS, $0.1 \%(\mathrm{v} / \mathrm{v})$ Tween 20 and incubated for $1 \mathrm{~h}$ at RT in HRP-conjugated anti-rabbit (\#W4011) or anti-mouse (\#W4021) secondary antibodies (Promega) in 5\% (v/v) nonfat dry milk in TBS, $0.1 \%(\mathrm{v} / \mathrm{v})$ Tween 20 . The immunoblots were developed using the ECL detection reagent (RPN2106, Amersham Biosciences).

\section{Cytosol and membrane fractionation}

Cells were washed twice in PBS and homogenized in homogenization buffer (150 mM NaCl, $1 \mathrm{mM}$ EGTA, 0.1 $\mathrm{mM} \mathrm{MgCl} 2,10 \mathrm{mM}$ HEPES, pH 7.4) containing phosphatase and protease inhibitors $(2 \mathrm{mM}$ sodium orthovanadate, $10 \mathrm{mM} \mathrm{NaF}, 1 \mathrm{mM}$ tetrasodium pyrophosphate, $10 \mu \mathrm{g} / \mathrm{ml}$ leupeptin, $250 \mu \mathrm{M}$ PMSF) and subjected to subcellular fractionation as described previously (Yu et al., 1999; Ha and Pak, 2005) with the following modification. Briefly, cell homogenates were centrifuged for $5 \mathrm{~min}$ at $200 \mathrm{~g}$ to remove nuclei and the resulting supernatant was recentrifuged at $16,000 \mathrm{~g}$ for $15 \mathrm{~min}$ yielding a pellet of plasma membrane containing membrane fraction. The supernatant representing cytosolic fraction was concentrated. The membrane and cytosolic fractions were subject to immunoblot analysis with anti-caveolin-3 antibody.

\section{Purification of caveolin-rich membrane fractions}

Isolation of caveolae was performed without detergent as described previously (Smart et al., 1995; Ha and Pak, 2005; Head et al., 2006; Kang et al., 2006). Briefly, cells were harvested with $0.5 \mathrm{M}$ sodium carbonate, $\mathrm{pH} 11.0$ at $4^{\circ} \mathrm{C}$. To disrupt cellular membranes, homogenization was carried out with a loose fitting Dounce homogenizer (10 strokes). The homogenate was then adjusted to $40 \%$ sucrose by adding an equal volume of $80 \%$ sucrose prepared in Mes-buffer saline (25 mM Mes, pH 6.5, $150 \mathrm{mM} \mathrm{NaCl}$ ), placed on the bottom of an ultracentrifuge tube, overlaid with a $5-35 \%$ discontinuous sucrose gradient $(4 \mathrm{ml}$ of $5 \%$ sucrose, $4 \mathrm{ml}$ of $35 \%$ sucrose; both in Mes-buffered saline containing $250 \mathrm{mM}$ sodium carbonate), and centrifuged at $39,000 \mathrm{~g}$ for $18 \mathrm{~h}$ in a SW41 rotor (Beckman Instruments, Palo Alto, CA). After the centrifugation, twelve 1-ml gradient fractions and a pellet were collected. Caveolar fractions were routinely localized in fractions 4,5 and 6 . Each 1-ml fraction was concentrated by precipitation with $20 \%$ TCA for $30 \mathrm{~min}$ in ice. Precipitated proteins were pelleted by centrifugation at $3,700 \mathrm{~g}$ for $15 \mathrm{~min}$ at $4^{\circ} \mathrm{C}$. The pellets were washed twice with $80 \%$ ethanol and dissolved in SDS-PAGE sample buffer for SDS-PAGE and immunoblotting for caveolin-3. 


\section{Mitochondria fractionation}

Cells were incubated with $50 \mu \mathrm{M}$ PE for $48 \mathrm{~h}$ or $10 \mu \mathrm{M}$ ISO for $48 \mathrm{~h}$ and subjected to subcellular fractionation (Potla et al., 2006). Cells were harvested by centrifugation, washed with PBS, and resuspended in ice-cold P21 buffer $(5 \mu \mathrm{g} / \mathrm{ml}$ aprotinin, $1 \mu \mathrm{g} / \mathrm{ml}$ leupeptin, $25 \mu \mathrm{g} / \mathrm{ml}$ perfabloc, $1 \mu \mathrm{g} / \mathrm{ml}$ pepstatin A, $200 \mu \mathrm{M}$ PMSF, and $1 \mathrm{mM}$ sodium orthovanadate). The cells were homogenized and centrifuged at $1,000 \mathrm{rpm}$ for $5 \mathrm{~min}$ at $4^{\circ} \mathrm{C}$ to pellet nuclei as nuclear fraction. The supernatant was centrifuged at $8,700 \mathrm{rpm}$ for $10 \mathrm{~min}$ at $4^{\circ} \mathrm{C}$. The supernatant was then transferred to a fresh tube and centrifuged at $46,000 \mathrm{rpm}$ for $1 \mathrm{~h}$ at $4^{\circ} \mathrm{C}$ (Sorvall Ultra microcentrifuge, Rotor: S45A). The resulting supernatant, cytosolic fraction was collected. The pellet was resuspended using approximately $70-100 \mu$ fresh P21 buffer and layered on the top of a sucrose-Percoll gradient and centrifuged at $27,000 \mathrm{rpm}$ for $45 \mathrm{~min}$ at $4^{\circ} \mathrm{C}$ (Sorvall Ultra microcentrifuge, Rotor: S45A). The mitochondrial pellet was appeared in the middle of the tube. The mitochondrial pellet was resuspended with $1 \mathrm{ml}$ of a 50:50 mix of P21 buffer containing sucrose and EDTA and P21 buffer without sucrose and EDTA and centrifuged at $8,700 \mathrm{rpm}$ for $10 \mathrm{~min}$ at $4^{\circ} \mathrm{C}$. The pellet was taken and added $100 \mu \mathrm{l}$ of P21 buffer (containing sucrose and EDTA) plus 1\% CHAPS to solubilize the pellet, and incubated on ice for $15 \mathrm{~min}$. The solubilized mitochondrial fraction was centrifuged at 14,000 rpm for 10 min at $4^{\circ} \mathrm{C}$. The supernatant was finally collected as mitochondrial fraction. The nuclear, cytosolic and mitochondrial fractions were subjected to immunoblot analysis with anti-STAT3, anti-pS727-STAT3, anti-pY705STAT3, and anti-complex II, III, and V antibodies.

\section{Immunofluorescence microscopy}

$\mathrm{H} 9 \mathrm{c} 2$ cells grown on glass coverslips were serum starved for $18 \mathrm{~h}$ in DMEM containing 1\% FBS and treated with either PE or ISO for $48 \mathrm{~h}$. The cells were washed with PBS and fixed for 20 min at RT with $3.7 \%$ paraformaldehyde in PBS. Fixed cells were rinsed with PBS and permeabilized with PBS containing $0.1 \%$ Triton X-100 for 20 min. Permeabilized cells were rinsed with PBS, incubated with $1 \%$ BSA in PBS for 30 min and then with anti-caveolin-3 and anti-pY705-STAT3 antibodies diluted $1 / 500$ in 1\% BSA in PBS for $2 \mathrm{~h}$ at RT. After washing three times with PBS, the primary antibodies were detected with Alexa Fluor ${ }^{\mathbb{R}} 488$ conjugated anti-mouse (1/250 dilution) (Invitrogen) or TRITC-conjugated anti-mouse (1/200 dilution) IgG antibodies (Jackson ImmunoResearch Laboratories Inc) for caveolin-3 and pY705-STAT3, respectively for $1 \mathrm{~h} \mathrm{RT}$. The coverslips were then washed and mounted on glass slides. Fluorescent images were obtained using appropriate filters on an Olympus BX51 microscope and imaged with an Olympus DP-71 digital camera with an image processing system equipped with Image-ProPlus 6.1 (MediaCybernetics) as described previously (Ha and Pak, 2005; Kwon et al., 2008). Neither labeling in the absence of the primary antibody nor cross-reactivity between secondary and primary antibodies was observed.

\section{Densitometry analysis}

Autoradiograms were quantified with an imaging den- sitometer using the Molecular Analyst software (Bio-Rad Laboratories, model GS-700). Signal intensities of bands in immunoblots were determined by the scanning laser densitometry.

\section{Statistical analysis}

Values are means \pm S.E. Results were analyzed using the student's $t$-test. $P<0.05$ was considered indicative of a significant difference.

\section{Acknowledgments}

This work was supported in part by the fund of Research Promotion Program (RPP-2006-020), Gyeongsang National University and by a grant from the MOST/KOSEF to the EBNCRC to YP. HK, KJ, and CM were supported by a scholarship from the BK21 Program, the Ministry of Education and Human Resources Development, Korea.

\section{References}

Alvarez BV, Johnson DE, Sowah D, Soliman D, Light PE, Xia Y, Karmazyn M, Casey JR. Carbonic anhydrase inhibition prevents and reverts cardiomyocyte hypertrophy. J Physiol 2007;579:127-45

Anderson RG. The caveolae membrane system. Annu Rev Biochem 1998;67:199-225

Colombo F, Noël J, Mayers P, Mercier I, Calderone A. Beta-adrenergic stimulation of rat cardiac fibroblasts promotes protein synthesis via the activation of phosphatidylinositol 3-kinase. J Mol Cell Cardiol 2001;33:1091-106

Couet J, Sargiacomo M, Lisanti MP. Interaction of a receptor tyrosine kinase, EGF-R, with caveolins. Caveolin binding negatively regulates tyrosine and serine/threonine kinase activities. J Biol Chem 1997;272:30429-38

Dorn GW 2nd, Force T. Protein kinase cascades in the regulation of cardiac hypertrophy. J Clin Invest 2005;115: 527-37

Everson WV, Smart EJ. Influence of caveolin, cholesterol, and lipoproteins on nitric oxide synthase implications for vascular disease. Trends Cardiovasc Med 2001;11:246-50

Fanzani A, Musarò A, Stoppani E, Giuliani R, Colombo F, Preti A, Marchesini S. Hypertrophy and atrophy inversely regulate caveolin-3 expression in myoblasts. Biochem Biophys Res Commun 2007;357:314-8

$\mathrm{Ha} \mathrm{H}$, Pak Y. Modulation of the caveolin-3 and Akt status in caveolae by insulin resistance in $\mathrm{H} 9 \mathrm{c} 2$ cardiomyoblasts. Exp Mol Med 2005;37:169-78

Head BP, Patel HH, Roth DM, Murray F, Swaney JS, Niesman $I R$, Farquhar MG, Insel PA. Microtubules and actin microfilaments regulate lipid raft/caveolae localization of adenylyl cyclase signaling components. J Biol Chem 2006;281:26391-9

Hwang GS, Oh KS, Koo HN, Seo HW, You KH, Lee BH. Effects of KR-31378, a novel ATP-sensitive potassium 
channel activator, on hypertrophy of $\mathrm{H} 9 \mathrm{c} 2$ cells and on cardiac dysfunction in rats with congestive heart failure. Eur J Pharmacol 2006;540:131-8

Kang MJ, Chung $\mathrm{YH}$, Hwang $\mathrm{Cl}$, Murata M, Fujimoto T, Mook-Jung $\mathrm{IH}$, Cha $\mathrm{Cl}$, Park WY. Caveolin-1 upregulation in senescent neurons alters amyloid precursor protein processing. Exp Mol Med 2006;38:126-33

Karpen JW, Rich TC. The fourth dimension in cellular signaling. Science 2001;293:2204-5

Kato T, Sano M, Miyoshi S, Sato T, Hakuno D, Ishida H, Kinoshita-Nakazawa H, Fukuda K, Ogawa S. Calmodulin kinases II and IV and calcineurin are involved in leukemia inhibitory factor-induced cardiac hypertrophy in rats. Circ Res 2000;87:937-45

Kim S, Pak Y. Caveolin-2 regulation of the cell cycle in response to insulin in Hirc-B fibroblast cells. Biochem Biophys Res Commun 2005;330:88-96

Kunisada K, Tone E, Fujio Y, Matsui H, Yamauchi-Takihara $\mathrm{K}$, Kishimoto T. Activation of gp130 transduces hypertrophic signals via STAT3 in cardiac myocytes. Circulation 1998;98:346-52

Kunisada K, Negoro S, Tone E, Funamoto M, Osugi T, Yamada S, Okabe M, Kishimoto T, Yamauchi-Takihara K. Signal transducer and activator of transcription 3 in the heart transduces not only a hypertrophic signal but a protective signal against doxorubicin-induced cardiomyopathy. Proc Natl Acad Sci USA 2000;97:315-9

Kwon H, Jeong K, Pak Y. Identification of pY19-caveolin-2 as a positive regulator of insulin-stimulated actin cytoskeletondependent mitogenesis. J Cell Mol Med 2008; doi:10.1111/ j.1582-4934.2008.00391.

Li H, Brodsky S, Basco M, Romanov V, De Angelis DA, Goligorsky MS. Nitric oxide attenuates signal transduction: possible role in dissociating caveolin-1 scaffold. Circ Res 2001;88:229-36

Liu J, Razani B, Tang S, Terman BI, Ware JA, Lisanti MP. Angiogenesis activators and inhibitors differentially regulate caveolin-1 expression and caveolae formation in vascular endothelial cells. Angiogenesis inhibitors block vascular endothelial growth factor-induced down-regulation of caveolin-1. J Biol Chem 1999;274:15781-5

Lufei C, Ma J, Huang G, Zhang T, Novotny-Diermayr V, Ong CT, Cao X. GRIM-19, a death-regulatory gene product, suppresses Stat3 activity via functional interaction. EMBO J 2003;22:1325-35

Nicol RL, Frey N, Pearson G, Cobb M, Richardson J, Olson EN. Activated MEK5 induces serial assembly of sarcomeres and eccentric cardiac hypertrophy. EMBO J 2001;20: 2757-67

Oh $P$, Schnitzer JE. Segregation of hetero-trimeric $G$ proteins in cell surface microdomains. $G(q)$ binds caveolin to concentrate in caveolae, whereas $\mathrm{G}(\mathrm{i})$ and $\mathrm{G}(\mathrm{s})$ target lipid rafts by default. Mol Biol Cell 2001;12:685-98

Potla R, Koeck T, Wegrzyn J, Cherukuri S, Shimoda K, Baker
DP, Wolfman J, Planchon SM, Esposito C, Hoit B, Dulak J, Wolfman A, Stuehr D, Larner AC. Tyk2 tyrosine kinase expression is required for the maintenance of mitochondrial respiration in primary pro-B lymphocytes. Mol Cell Biol 2006;26:8562-71

Razani B, Woodman SE, Lisanti MP. Caveolae: from cell biology to animal physiology. Pharmacol Rev 2002;54: 431-67

Sano M, Fukuda K, Kodama H, Pan J, Saito M, Matsuzaki J, Takahashi T, Makino S, Kato T, Ogawa S. Interleukin-6 family of cytokines mediate angiotensin II-induced cardiac hypertrophy in rodent cardiomyocytes. J Biol Chem 2000;275:29717-23

Schägger $\mathrm{H}$, Pfeiffer K. Supercomplexes in the respiratory chains of yeast and mammalian mitochondria. EMBO J 2000;19:1777-83

Schlegel A, Schwab RB, Scherer PE, Lisanti MP. A role for the caveolin scaffolding domain in mediating the membrane attachment of caveolin-1. The caveolin scaffolding domain is both necessary and sufficient for membrane binding in vitro. J Biol Chem 1999;274:22660-7

Segal SS, Brett SE, Sessa WC. Codistribution of NOS and caveolin throughout peripheral vasculature and skeletal muscle of hamsters. Am J Physiol 1999;277:H1167-H77

Smart EJ, Ying YS, Mineo C, Anderson RG. A detergent-free method for purifying caveolae membrane from tissue culture cells. Proc Natl Acad Sci USA 1995;92:10104-8

Smart EJ, Graf GA, McNiven MA, Sessa WC, Engelman JA, Scherer PE, Okamoto T, Lisanti MP. Caveolins, liquidordered domains, and signal transduction. Mol Cell Biol 1999;19:7289-304

Taigen T, De Windt LJ, Lim HW, Molkentin JD. Targeted inhibition of calcineurin prevents agonist-induced cardiomyocyte hypertrophy. Proc Natl Acad Sci USA 2000;97: 1196-201

Takeo S, Elmoselhi AB, Goel R, Sentex E, Wang J, Dhalla NS. Attenuation of changes in sarcoplasmic reticular gene expression in cardiac hypertrophy by proparanolol and verapamil. Mol Cell Biochem 2000;213:111-8

Watanabe T, Pakala R, Katagiri T, Benedict CR. Angiotensin II and serotonin potentiate endothelin-1-induced vascular smooth muscle cell proliferation. J Hypertens 2001;19:731-9

Yamamoto M, Toya Y, Jensen RA, Ishikawa Y. Caveolin is an inhibitor of platelet-derived growth factor receptor signaling. Exp Cell Res 1999;247:380-8

Yu B, Poirier LA, Nagy LE. Mobilization of GLUT4 from intracellular vesicles by insulin and $\mathrm{K}(+)$ depolarization in cultured H9c2 myotubes. Am J Physiol 1999;277:E259- E67

Zampaglione B, Pascale C, Marchisio M, Cavallo-Perin P. Hypertensive urgencies and emergencies. Prevalence and clinical presentation. Hypertension 1996;27:144-7

Zhang H, Feng W, Liao W, Ma X, Han Q, Zhang Y. The gp130/STAT3 signaling pathway mediates beta-adrenergic receptor-induced atrial natriuretic factor expression in 
cardiomyocytes. FEBS J 2008;275:3590-7

Zhang J, Yang J, Roy SK, Tininini S, Hu J, Bromberg JF, Poli V, Stark GR, Kalvakolanu DV. The cell death regulator GRIM-19 is an inhibitor of signal transducer and activator of transcription 3. Proc Natl Acad Sci USA 2003;100:9342-7
Zou Y, Yao A, Zhu W, Kudoh S, Hiroi Y, Shimoyama M, Uozumi H, Kohmoto O, Takahashi T, Shibasaki F, Nagai R, Yazaki Y, Komuro I. Isoproterenol activates extracellular signal-regulated protein kinases in cardiomyocytes through calcineurin. Circulation 2001;104:102-8 